\title{
On the Classical Limit of Berry's Phase Integrable Systems
}

\author{
J. Asch \\ Technische Universität Berlin, Fachbereich Mathematik, Ma7-1, \\ Straße des 17. Juni 136, D-1000 Berlin 12
}

\begin{abstract}
Berry's Phase is given by integration of a characteristic two form. We consider integrable systems defined by Weyl quantized classical Hamiltonians. It is shown that the limit of $\hbar / i$ times this two form is the curvature of the classical connection whose holonomy is the Hannay angles. A result of this type was derived by Berry [B2].
\end{abstract}

\section{Introduction}

Consider a quantum system whose dynamics is determined by a family of selfadjoint Hamiltonians on a Hilbert space depending smoothly on several parameters. Consider furthermore a region of energies such that the corresponding spectral subspace - defined by a projection $P$ - also varies in a smooth manner.

Thus a vector bundle over the manifold of parameters is defined. As it is embedded in the trivial Hilbert bundle $P d$ is a natural connection on it $-d$ denotes differentiation with respect to the parameters. It is well known [K], [A-S-Y] that as the parameters are driven adiabatically slowly in time the dynamical evolution starting in the spectral subspace is essentially the parallel - adiabatic - movement induced by this connection.

Curvature and holonomy effects have gained considerable interest in chemistry and physics. This was initiated by the work of Mead-Truhlar [M-T], Berry $[\mathrm{B}]$ and Simon $[S]$ who first recognized the geometrical meaning.

We are interested in the quantum two form tr $P d P d P$ which represents the first Chern class of the bundle. Integrated over the interior of a contractible loop it gives Berry's Phase in the case of a line bundle.

Let the operators be given by quantization of classical Hamiltonians. All objects then depend on a semiclassical parameter $\hbar$. Consider a sequence of eigenvalues approaching a given classical energy as $\hbar$ goes to zero and the corresponding eigenprojections $P$. 
Our question is:

Is there a limit of $\operatorname{tr} P d P d P$ as $\hbar$ goes to zero and how can this be interpreted geometrically?

We proceed as follows: If the classical flow is ergodic on the energy shell it is known [H-M-R] that - morally - the semiclassical limit of $\operatorname{tr} P A$ is $\langle a\rangle$ for a PseudoDifferential Operator ( $\psi \mathrm{DO}) A$ with symbol $a,\langle\cdot\rangle$ denoting the mean over the energy shell. So we should like to approximate $d P$ in some sense by a $\psi \mathrm{DO}$.

This can be achieved with an operator whose symbol solves an equation which is - morally $-\{\cdot, f(h)\}=d f(h)$, where $h$ is the classical Hamiltonian and $f$ a suitable function.

As it is not clear whether such an equation admits smooth solutions for general ergodic systems we are fonced to restrict ourselves to the integrable case which is ergodic in the sense that the joint flow of the commuting constants of motion is ergodic on the joint energy torus. Here we can solve the equation for the symbol and use the solution for the computation of the limit of the quantum two form.

In geometric terms our result may be described as follows: For integrable systems a manifold of fixed actions in parameter-phase space is a torus bundle on which a natural connection is given. This was recently shown by $[\mathrm{M}],[\mathrm{Kn}]$.

We shall show that $\operatorname{tr} P d P d P$ multiplied by $\hbar / i$ tends to the curvature of this connection for $\hbar \rightarrow 0$. Here $P$ is the projection on the joint energy space of $n$ commuting Hamiltonians $-n$ the number of freedoms. A result of this type was derived by Berry [B2] in a special coordinate system.

For more general systems one might define a smoothed out quantum two form which can be expanded asymptotically in $\hbar$ without any assumptions on the classical flow. This was suggested by [Se] and [S-T].

In the next section we shall state the assumptions on the system and describe the strategy. In Sect. 2 a differentiable functional calculus for $\psi$ DOs will be developed and the classical equation will be solved. Section 3 is dedicated to the proof and interpretation of the results for integrable systems. Finally in Sect. 4 we prove that the smoothed out quantum two form admits an asymptotic expansion in $\hbar$.

\section{Assumptions and Strategy}

By quantization of Hamiltonian functions we want to obtain a family of selfadjoint operators which is differentiable in norm-resolvent sense. This is assured by the first two sets of hypotheses.

For a positive continuous function $w$ on $\mathbb{R}^{2 n}$ denote

$$
S(w):=\left\{f \in C^{\infty}\left(\mathbb{R}^{2 n}\right) ; \quad\|f\|_{w, \alpha}:=\sup _{\mathbb{R}^{2 n}}\left|\frac{D^{\alpha} f}{w}\right|<\infty \quad\left(\alpha \in \mathbb{N}_{0}^{2 n}\right)\right\} .
$$

$S(w)$ is a Fréchet space in the topology defined by the seminorms $\|\cdot\|_{w, \alpha}$.

Let $n, d$ be elements of $\mathbb{N}, U_{0} \subset \mathbb{R}^{d}$ be an open ball around zero. A function $h: U_{0} \times \mathbb{R}^{2 n} \rightarrow \mathbb{R}$ satisfies the conditions (H1) iff: 
(i) $h(\kappa) \in C^{\infty}\left(\mathbb{R}^{2 n}\right) \quad\left(\kappa \in U_{0}\right)$,

(ii) $h(\kappa, p)>\gamma \quad\left(\right.$ for $\left.a \gamma>0,(\kappa, p) \in U_{0} \times \mathbb{R}^{2 n}\right)$,

(iii) for $\kappa \in U_{0} \quad \exists c>0, N \in \mathbb{N}$ such that $h(\kappa, p) \leqq h(\kappa, q)(1+|p-\mathrm{q}|) \mathcal{N}$ for all $p, q \in \mathbb{R}^{2 n}$,

(iv) $h(\kappa) \in S(h(\kappa))\left(\kappa \in U_{0}\right)$.

For quantization we use the Weyl scheme: For $\psi \in \mathscr{S}\left(\mathbb{R}^{n}\right), \hbar \in(0,1), \kappa \in U_{0}$,

$$
h(\kappa)(x, \hbar D) \psi(x):=\int_{\mathbb{R}^{n}} \int_{\mathbb{R}^{n}} \exp \left(\frac{i}{\hbar}(x-y, \xi)\right) h\left(\kappa, \frac{x+y}{2}, \xi\right) \psi(y) \frac{d y d \xi}{(2 \pi \hbar)^{n}} .
$$

The following statement is known cf. [H-R]:

Theorem 1. Let h satisfy (H1), then

$$
h(\kappa)(x, \hbar D) \text { is essentially selfadjoint for } \kappa \in U_{0} .
$$

Suppressing the dependence upon $\hbar$ we denote the closure by $H(\kappa)$.

Conditions $(\mathbf{H 2})$ are in addition to $(\mathrm{H} 1)$ :

(i) $\frac{h(\kappa, p)}{h(0, p)} \geqq c>0 \quad\left(\right.$ some $\left.c,(\kappa, p) \in U_{0} \times \mathbb{R}^{2 n}\right)$,

(ii) $h \in C^{1}\left(U_{0}, S(h(0, \cdot))\right)$.

They are sufficient for differentiability:

Theorem 2. Let h satisfy (H1), (H2). For $\forall \varepsilon>0 \exists \eta \in(0,1) \forall \hbar<\eta \forall z \in \mathbb{C} \backslash[\gamma-\varepsilon, \infty)$ the following holds:

(i) $z \in \varrho(H(\kappa))\left(\kappa \in U_{0}\right) \quad$ (* resolvent set $\left.*\right)$.

(ii) $(H(\cdot)-z)^{-1} \in C^{1}\left(U_{0}, B\left(L^{2}\left(\mathbb{R}^{n}\right)\right)\right)$.

(iii) There exist $r_{j}(z, \cdot) \in C^{1}\left(U_{0}, S(1)\right)\left(j \in \mathbb{N}_{0}\right)$ such that for $N \in \mathbb{N}_{0}$

$$
(H(\cdot)-z)^{-1}=\sum_{j=0}^{N} \overline{\hbar^{j}} \overline{r_{j}(z, \cdot)(x, \hbar D)}+\hbar^{N+1} \mathcal{O}(1)
$$

in $U_{0} . \mathcal{O}(1)$ is $C^{1}\left(U_{0}, B\left(L^{2}\right)\right)$ uniformly in $\hbar$.

Proof. We get this extending the construction of the parametrix used in [H-R] to the parameter dependent case. Recall the definition of the \# operation [Hör]:

$$
\#: S\left(w_{1}\right) \times S\left(w_{2}\right) \rightarrow S\left(w_{1} w_{2}\right)
$$

is a continuous bilinear map with the property

$$
a_{1} \# a_{2}(x, \hbar D)=a_{1}(x, \hbar D) a_{2}(x, \hbar D) .
$$

For $N \in \mathbb{N}_{0}$ exists $s_{N} \in S\left(w_{1} w_{2}\right)$ such that

$$
\begin{aligned}
a_{1} \# a_{2}(x, \xi)= & \left.\sum_{j=0}^{N} \frac{1}{j !}\left(\frac{\hbar i}{2} \sigma\left(D_{x}, D_{\xi} ; D_{y}, D_{\eta}\right)\right)^{j} a_{1}(x, \xi) a_{2}(y, \eta)\right|_{(x, \xi)=(y, \eta)} \\
& \hbar^{N+1} S_{N}\left((x, \xi) \in \mathbb{R}^{2 n}\right),
\end{aligned}
$$

where $\sigma(x, \xi ; y, \eta):=\langle\xi, x\rangle-\langle\eta, y\rangle \quad\left((x, \xi),(y, \eta) \in \mathbb{R}^{2 n}\right)$. 
Define $r_{j}(z, \kappa) \in S(1 / h(\kappa))$ such that

$$
\begin{aligned}
(h-z)(x, \hbar D) \sum_{j=0}^{N} \hbar^{j} r_{j}(z)(x, \hbar D) & =\left(h-\mathrm{z} \# \sum_{j=0}^{N} \hbar^{j} r_{j}(z)\right)(x, \hbar D) \\
& =1+\hbar^{N+1} \delta_{N}(z, \hbar)(x, \hbar D)
\end{aligned}
$$

with $\delta_{N}(z, \hbar) \in S(1), \sup _{\hbar}\left\|\delta_{N}(z, \hbar)(x, \hbar D)\right\|<\infty$.

Then $r_{1} \equiv 0$ by construction, $z \in \varrho(H(\kappa))$ and

$$
(H(\cdot)-z)^{-1}=\sum_{j=0}^{N} \hbar^{j} \overline{r_{j}(z, \cdot)(x, \hbar D)}-\hbar^{N+1}(H(\cdot)-z)^{-1} \overline{\delta_{N}(z, \hbar, \cdot)(x, \hbar D)} .
$$

From the explicit form of $r_{j}$ and as \# is bilinear and continuous one sees: $r_{j} \in C^{1}\left(U_{0}, S(1 / h(0)), \delta_{N} \in C^{1}\left(U_{0}, S(1)\right)\right.$.

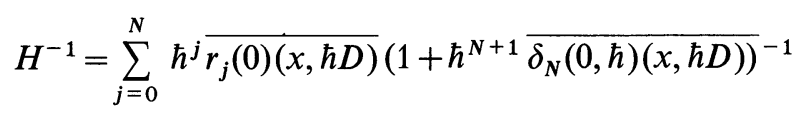

implies $H^{-1} \in C^{1}\left(U_{0}, B\left(L^{2}\right)\right)$ uniformly in $\hbar<\eta$.

For $0 \neq z \in \varrho(H)$ differentiability of $(H(\cdot)-z)^{-1}$ and $\mathcal{O}(1)$ follow from

$$
(H-z)^{-1}=-\frac{1}{z}-\frac{1}{z^{2}}\left(H^{-1}-\frac{1}{z}\right)^{-1} \text {. }
$$

Let us now state the problem more precisely.

For $n$ functions $h_{v}$ satisfying $(\mathrm{H} 1)$, (H2) suppose $(\mathbf{H 3})$ :

$$
\left[\left(H_{\lambda}(\kappa)-z_{\lambda}\right)^{-1},\left(H_{v}(\kappa)-z_{v}\right)^{-1}\right]=0 \quad\left(\text { all } \lambda, v, \kappa \in U_{0}, z_{\lambda, v} \in \varrho\left(H_{\lambda, v}(\kappa)\right)\right) .
$$

Fix a classical "energy" $E_{0} \in \mathbb{R}^{n}$ and consider sequences $E_{\hbar}^{v} \in \sigma_{d}\left(H_{v}(0)\right.$ ) (* discrete $\operatorname{spectrum} *)$ with $\left\langle E_{\hbar}^{1}, \ldots, E_{\hbar}^{n}\right\rangle \rightarrow E_{0}(\hbar \rightarrow 0)$.

By Theorem 2 the corresponding eigenprojections $P_{v}^{\hbar}$ are $C^{1}$ as bounded operators for every $\hbar$ in a vicinity $V_{\hbar}$ of 0 . So is $P_{\hbar}:=\prod_{v=1}^{n} P_{v}^{\hbar}$, which by the commutativity assumption is the projection on the common eigenspace. We are interested in the limit as $\hbar$ tends to zero of

$$
\left.\operatorname{tr} P_{\hbar} d P_{\hbar} d P_{\hbar}\right|_{\kappa=0} \text {. }
$$

In order to apply $\psi \mathrm{DO}$ techniques the operator in $\operatorname{tr} P(\cdot)$ has to be regularized. We shall suppress the index $\hbar$.

To illustrate our strategy we remark that $[d P, P]$ solves

$$
d P=[\cdot, P],
$$

which implies for its (pathwise) flow $U, U(0)=1$ :

$$
U(\kappa) P(0)=P(\kappa) U(\kappa) .
$$

Hence the physical significance of $[d P, P]$ is to generate adiabatic motion.

We shall demonstrate the following points: Firstly that a generator of classical adiabatic motion (in a sense to be explained) exists for our systems, secondly that the 
operator obtained by quantization solves the commutator equation up to an error small in $\hbar$, and finally that this is sufficient for the solution of our problem.

To do this we employ the following useful representation of $d P$ which we state without proof.

Lemma 3. Let $H$ be selfadjoint on a Hilbert space, $A \subset \sigma(H)$ separated by a nice closed curve $\Gamma$ from $\sigma(H) \backslash A$. Let $g: \mathbb{R} \rightarrow \mathbb{R}$ be measurable such that there is a nice closed curve $\Gamma_{g}$ which separates $g(A)$ from $g(\sigma(H) \backslash A)$. Then

$$
P(A):=-\frac{1}{2 \pi i} \int_{\Gamma}(H-\mathrm{z})^{-1} d z=-\frac{1}{2 \pi i} \int_{\Gamma_{g}}(g(H)-z)^{-1} d z .
$$

Corollary 4. Suppose that $f: \mathbb{R} \rightarrow \mathbb{R}$ satisfies the $g$ conditions of Lemma 3 for all $\kappa \in U_{0}, \quad v \in\{1, \ldots, n\}, \quad A_{\kappa}^{v}=\left\{E_{\hbar}^{v}(\kappa)\right\}, \quad H=H_{v}(\kappa)$. If furthermore $f\left(H_{v}\right)$ is in $C^{1}\left(U_{0}, B\left(L^{2}\right)\right)$, then

$$
d P_{v}=\frac{1}{2 \pi i} \int_{\Gamma v}\left(f\left(H_{v}\right)-z\right)^{-1} d f\left(H_{v}\right)\left(f\left(H_{v}\right)-z\right)^{-1} d z
$$

The corresponding representation of $d P$ is obtained via the Leibnitz-rule.

A one form $M$ on $\mathbb{R}^{d}$ with values in the bounded operators on $L^{2}\left(\mathbb{R}^{n}\right)$ which satisfies

$$
d f\left(H_{v}\right)=\left[M, f\left(H_{v}\right)\right] \text { for all } v
$$

would fulfill as well

$$
d P_{v}=\left[M, P_{v}\right] \text { for all } v .
$$

It will be shown that it is in fact sufficient for the last relation to establish the first on the off-diagonal with respect to the decomposition $1=P_{v}+Q_{v}$, namely

$$
P_{v} d f\left(H_{v}\right) Q_{v}+Q_{v} d f\left(H_{v}\right) P_{v}=P_{v}\left[M, f\left(H_{v}\right)\right] Q_{v}+Q_{v}\left[M, f\left(H_{v}\right)\right] P_{v} .
$$

The Leibnitz-rule then implies that a simultaneous solution at $\kappa=0$ for all $v$ of

$$
\begin{aligned}
P_{1} \ldots\left(P_{v} d f\left(H_{v}\right) Q_{v}+Q_{v} d f\left(H_{v}\right) P_{v}\right) \ldots P_{n}= & P_{1} \ldots\left(P_{v}\left[\cdot, f\left(H_{v}\right)\right] Q_{v}\right. \\
& \left.+Q_{v}\left[\cdot, f\left(H_{v}\right)\right] P_{v}\right) \ldots P_{n}
\end{aligned}
$$

solves $d P=[\cdot, P]$ at $\kappa=0$.

It will be sufficient for the solution of our problem to find such a one form with regular values which solves $(*)$ up to a semiclassical small error. In order to find this we will solve the classical equations corresponding to $(*)$ and quantize the solution.

\section{Differentiable Functional Calculus and the Classical Equation}

We shall develop a suitable tool for the semiclassical analysis and solve the classical equations corresponding to $(*)$. For $r \in \mathbb{R}$ denote

$$
\begin{aligned}
& S_{r}:=\left\{f \in C^{\infty}(\mathbb{R}) ; \operatorname{supp} f \subset(0, \infty),\right. \\
& \left.\|f\|^{(k)}:=\sup _{E \in \mathbb{R}}\left|(1+E)^{k-r} f^{(k)}(E)\right|<\infty,\left(k \in \mathbb{N}_{0}\right)\right\},
\end{aligned}
$$

which if equipped with its seminorms is a Fréchet space. 
Theorem 5. Let $f$ be in $C^{1}\left(U_{0}, S_{r}\right)$ for an $r<-2$, assume (H1), (H2) on $h$. Then

(i) $f(\cdot, H(\cdot)) \in C^{1}\left(U_{0}, B\left(L^{2}\right)\right)$.

(ii) There exist $f_{j}(\cdot) \in C^{1}\left(U_{0}, S(1)\right)\left(j \in \mathbb{N}_{0}\right)$ such that for $N \in \mathbb{N}_{0}$,

$$
f(\kappa, H(\kappa))=\sum_{j=0}^{N} \overline{\hbar^{j}} \overline{f_{j}(\kappa)(x, \hbar D)}+\hbar^{N+1} \mathcal{O}(1)
$$

with $\mathcal{O}(1) \in C^{1}\left(U_{0}, B\left(L^{2}\right)\right)$ uniformly in $\hbar$.

(iii) $f_{0}(\kappa, p)=f(\kappa, h(\kappa, p)), f_{1} \equiv 0, d f_{j}(x, \hbar D)=\left(d f_{j}\right)(x, \hbar D)$

(*d always denotes the differential with respect to $\kappa *$ ).

Proof. Again this is by extension of results of [H-R] to parameter dependence. We give an outline of the ideas. Denote

$$
M f(s, \kappa):=\int_{0}^{\infty} E^{s-1} f(\kappa, E) d E \quad(s \in \varrho+i \mathbb{R}, \varrho \in(0,-r))
$$

the Mellin transformation of $f$. Mellin inversion formula implies via the spectral calculus

$$
f(\kappa, H(\kappa))=-\frac{1}{2 \pi i} \int_{\varrho+i \mathbb{R}} M f(s, \kappa) H^{-s}(\kappa) d s \quad(\varrho \in(0,-r)) .
$$

$M f$ is $C^{1}$ with respect to $\kappa$ in each of the norms given by

$$
\sup _{s \in \varrho+i \mathbb{R}}\left|\left(1+|\operatorname{Im} s|^{k}\right) M f(s)\right| \quad \text { for } k \in \mathbb{N}_{0}
$$

$H^{-s}$ has the representation

$$
H^{-s}(\kappa)=-\frac{1}{2 \pi i} \int_{\Gamma_{\theta}} z^{-s}(H(\kappa)-z)^{-1} d z
$$

for any conical curve $\Gamma_{\theta}$ with opening angle $2 \theta$ surrounding the spectrum of $H(\kappa)$ in the right half plane.

Using the identity

$$
(H-z)^{-1}=-\frac{1}{z}-\frac{1}{z^{2}}\left(H^{-1}-\frac{1}{z}\right)^{-1} \quad(z \in \varrho(H) \backslash\{0\})
$$

and Theorem 2 one deduces that $H^{-s}(\cdot)$ is $C^{1}$ in the norm

$$
\sup _{s \in \varrho+i \mathbb{R}}\left(1+|\operatorname{Im} s|^{k}\right)^{-1} \| \text { Operator }(s) \|
$$

for $\varrho>2$ and some $k \in \mathbb{N}$.

Furthermore it follows from Theorem 2 that $\mathrm{H}^{-\mathrm{s}}$ has an asymptotic expansion by regular operators with Weyl symbols differentiable in the $S(1)$ norms weighted in the same manner. This implies the assertion.

The result carries over to functions $f\left(H_{1}, \ldots, H_{n}\right)$. We shall need only the following statement which we cite from [Ch]. 
Theorem 6. For $f \in C_{0}^{\infty}\left(\mathbb{R}^{n}\right)$ there exist $f_{j} \in S(1)\left(j \in \mathbb{N}_{0}\right)$ such that for $N \in \mathbb{N}_{0}$,

$$
f\left(H_{1}, \ldots, H_{n}\right)=\sum_{j=0}^{N} \hbar^{j} \overline{f_{j}(x, \hbar D)}+\hbar^{N+1} \mathcal{O}(1) .
$$

In particular $f_{0}(p)=f\left(h_{1}, \ldots, h_{n}\right)(p)\left(p \in \mathbb{R}^{2 n}\right), f_{1} \equiv 0$.

We are now able to attack equations $(*)$.

Weyl calculus implies that the first term in the asymptotic expansion of a commutator is the Poisson bracket. Thus the classical equations for $(*)$ are modulo terms constant on the energy torus -

$$
d f\left(h_{v}\right)=\left\{\cdot, f\left(h_{v}\right)\right\} \quad \text { at } \kappa=0 \quad(v \in\{1, \ldots, n\}) .
$$

If the classical system is integrable this system of PDEs can be solved.

Denote by $h$ the map $h: \mathbb{R}^{2 n} \rightarrow \mathbb{R}^{n}, h(p):=\left(h_{1}(p), \ldots, h_{n}(p)\right)$ at $\kappa=0$.

In addition to the assumptions already made we suppose (H4) at $\kappa=0$ :

$\nabla_{p} h_{1}, \ldots, \nabla_{p} h_{n}$ are linearly independent in a vicinity $V$ of $h^{-1}\left(E_{0}\right),\left.h\right|_{V}$ is proper,

$h^{-1}\left(E_{0}\right) \subset \mathbb{R}^{2 n}$ is connected.

(H3) implies $\left\{h_{\lambda}, h_{v}\right\}=0$ for all $\lambda, v$. By the Theorem of Liouville Arnol'd there are neighborhoods $U$ of $h^{-1}\left(E_{0}\right), U_{1}$ of $E_{0}$, an open set $U_{2} \subset \mathbb{R}^{n}$ and diffeomorphisms

such that

$$
\begin{gathered}
\phi: U_{1} \rightarrow U_{2}, \\
\psi: \mathbb{T}^{n} \times U_{2} \rightarrow U,
\end{gathered}
$$

$$
\psi^{*} d \xi_{i} \wedge d x^{i}=d y_{i} \wedge d x^{i}, \quad \phi \circ h \circ \psi(x, y)=y \quad\left((x, y) \in \mathbb{T}^{n} \times U_{2}\right) .
$$

Denote by $\phi_{v}$ the components of $\phi$ and by $\pi_{v}$ the flow of $\phi_{v} \circ h$. Then it holds for the joint flow $\pi(t, p):=\pi_{1}\left(t_{1}, \pi_{2}\left(t_{2}, \ldots, \pi_{n}\left(t_{n}, p\right)\right)\right)\left((t, p) \in \mathbb{R}^{n} \times U\right)$ :

$$
\begin{gathered}
\pi(t+\gamma, p)=\pi(t, p) \quad\left(\gamma \in \mathbb{Z}^{n}, t \in \mathbb{R}^{n}, p \in U\right) \\
\pi(t, p)=q \quad\left(p, q \in U \text { such that } h(p)=h(q), \text { a suitable } t \in \mathbb{T}^{n}\right) .
\end{gathered}
$$

For $f \in C^{\infty}(U)$ define the mean

$$
\langle f\rangle(y):=\int_{\mathbb{T}^{n}} f \circ \pi(t, \psi(x, y)) d t \quad\left(y \in U_{2} \text {, some } x \in \mathbb{T}^{n}\right)
$$

The solution of the classical equations will be consequent on

Lemma 7. Assume (H1),.., (H4). Consider $b_{\mu} \in C^{\infty}(U)(\mu \in\{1, \ldots, n\})$ with $\left\langle b_{\mu}\right\rangle \equiv 0$, $\left\{b_{\mu}, \phi_{v} \circ h\right\}=\left\{b_{v}, \phi_{\mu} \circ h\right\}(\mu, v \in\{1, \ldots, n\})$.

There exists an $a \in C^{\infty}(U)$ such that

$$
\left\{a, \phi_{\mu}^{\circ} h\right\}=b_{\mu} \quad(\mu \in\{1, \ldots, n\}) .
$$


Proof. Pick an $\omega \in \mathbb{R}^{n}$ such that for some norm on $\mathbb{R}^{n}$,

$$
|(\omega, k)| \geqq \gamma\|k\|^{-\sigma} \quad\left(k \in \mathbb{Z}^{n} \backslash\{0\}, \text { some } \gamma, \sigma>0\right),
$$

which exists by a theorem of Liouville.

Define for $\varepsilon>0$

$$
a_{\varepsilon}(p):=-\int_{0}^{\infty} e^{-\varepsilon s} \sum_{v=1}^{n} b_{v}(\pi(\omega s, p)) \omega_{v} d s \quad(p \in U) .
$$

Then $a_{\varepsilon} \in C^{\infty}(U)$ and

$$
\begin{aligned}
\left\{a_{\varepsilon}, \phi_{\mu} \circ h\right\}(p) & =-\int_{0}^{\infty} e^{-\varepsilon s} \sum_{v=1}^{n} \omega_{v}\left\{b_{v}, \phi_{\mu} \circ h\right\} \circ \pi(\omega s, p) d s \\
& =-\int_{0}^{\infty} e^{-\varepsilon s} \sum_{\nu=1}^{n} \omega_{v}\left\{b_{\mu}, \phi_{v} \circ h\right\} \circ \pi(\omega s, p) d s \\
& =-\int_{0}^{\infty} e^{-\varepsilon s} \frac{d}{d s} b_{\mu}(\pi(\omega s, p)) d s \\
& =b_{\mu}(p)+\varepsilon \int_{0}^{\infty} e^{-\varepsilon s} b_{\mu}(\pi(\omega s, p)) d s
\end{aligned}
$$

As $\pi$ is completely periodic we can handle the limit $\varepsilon \rightarrow 0$,

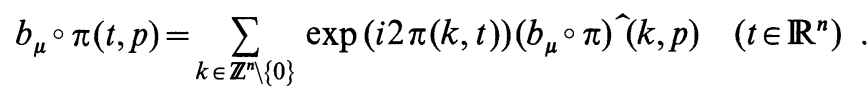

By smoothness

$$
\sup _{p \in \text { compact }}\left|\|k\|^{\beta} D_{p}^{\alpha}\left(b_{\mu}^{\circ} \pi\right)^{\wedge}(k, p)\right| \leqq \text { const } \quad\left(\alpha \in \mathbb{N}_{0}^{2 n}, \beta \in \mathbb{N}_{0}^{n}\right),
$$

which gives

$$
\begin{aligned}
D_{p}^{\alpha} \int_{0}^{\infty} e^{-\varepsilon s} b_{\mu}(\pi(\omega s, p)) d s= & \sum_{k \in \mathbb{Z}^{n} \backslash\{0\}} D_{p}^{\alpha}\left(b_{\mu}^{\circ} \pi\right) \hat{\gamma}(k, p) \int_{0}^{\infty} e^{(i 2 \pi(k, \omega)-\varepsilon) s} d s \\
= & \sum_{k \in \mathbb{Z}^{n} \backslash\{0\}} D_{p}^{\alpha}\left(b_{\mu} \circ \pi\right) \hat{(}(k, p) \frac{1}{\varepsilon-i 2 \pi(k, \omega)} \\
& \left(\alpha \in \mathbb{N}_{0}^{2 n}\right) .
\end{aligned}
$$

The diophantine condition on $\omega$ implies convergence uniformly in $\varepsilon$ for $p$ in compact sets. So

$$
a:=\lim _{\varepsilon \rightarrow 0} a_{\varepsilon} \in C^{\infty}(U), \quad D_{p}^{\alpha} a=\lim _{\varepsilon \rightarrow 0} D_{p}^{\alpha} a_{\varepsilon}
$$

Now follows

Corollary 8. There is a one form $\tilde{m}$ on $\mathbb{R}^{d}$ with values in $C^{\infty}(U)$ which satisfies $\left\{\tilde{m}, h_{\mu}\right\}=d h_{\mu}-\left\langle d h_{\mu}\right\rangle(\phi(h)) \quad$ in $U$ at $\kappa=0$ for all $\mu$. 
Proof. Define $b_{\mu}:=\sum_{\lambda=1}^{n}\left(\partial_{E^{\lambda}} \phi_{\mu}\right) \circ h\left(d h_{\lambda}-\left\langle d h_{\lambda}\right\rangle(\phi(h))\right)$. Then $b_{\mu}$ is a one form with values in $C^{\infty}(U),\left\langle b_{\mu}\right\rangle \equiv 0$,

$$
\left\{b_{\mu}, \phi_{v} \circ h\right\}=\sum_{\lambda, \varrho}\left(\partial_{E^{\lambda}} \phi_{\mu}\right)\left(\partial_{E^{e}} \phi_{v}\right) \circ h\left\{d h_{\lambda}, h_{\varrho}\right\}=\left\{b_{v}, \phi_{\mu} \circ h\right\}
$$

since $\left\{h_{\lambda}, h_{\varrho}\right\} \equiv 0$ in $U_{0} \times \mathbb{R}^{2 n}$.

Applying Lemma 7 to every component of the one form $b_{\mu}$ the corollary follows from the invertibility of $D_{E} \phi$.

We have accomplished the first step of the program stated in Sect. 1. Let us remark that it does not seem to be possible to find a smooth solution of the classical equations for general ergodic systems.

\section{Semiclassical Limit}

We shall show that the quantized solution of the classical equations obtained in Chapter 2 provides a regularization of $d P$ in the sense described in Sect. 1 and that this is sufficient for the computation of the limit of $\operatorname{tr} P d P d P$.

Clearly we can suppose $E_{0}=\left\langle e_{0}, \ldots, e_{0}\right\rangle$ for some $e_{0} \in(0, \infty)$ and furthermore for some $\varepsilon>0: U_{1}=\left(e_{0}-\varepsilon, e_{0}+\varepsilon\right) \times \ldots \times\left(e_{0}-\varepsilon, e_{0}+\varepsilon\right)$.

Let $f$ be in $C_{0}^{\infty}((0, \infty))$, decreasing in $(\gamma / 2, \infty)$, decreasing strictly in $\left(e_{0}-\varepsilon, e_{0}+\varepsilon\right)$. For $\eta>0$, let $\chi_{\eta}$ be in $C^{\infty}(\mathbb{R}), \chi_{\eta} \equiv 1$ in $\left(e_{0}-\eta / 2, e_{0}+\eta / 2\right), \chi_{\eta} \equiv 0$ in $\mathbb{R} \backslash\left(e_{0}-\eta, e_{0}+\eta\right)$

We now are able to give a regular approximate solution of $(*)$.

Theorem 9. Assume $(\mathrm{H} 1), \ldots,(\mathrm{H} 4)$. Then there is an $\eta>0$ and $a C_{0}^{\infty}\left(\mathbb{R}^{2 n}\right)$ valued one form $m$ on $\mathbb{R}^{d}$ such that for all $v \in\{1, \ldots, n\}$ the following holds at $\kappa=0$ :

(i) $\chi_{\eta}\left(h_{1}\right) \ldots \chi_{\eta}\left(h_{n}\right)\left\{m, f\left(h_{v}\right)\right\}=\chi_{\eta}\left(h_{1}\right) \ldots \chi_{\eta}\left(h_{n}\right)\left(d f\left(h_{v}\right)-\left\langle d f\left(h_{v}\right)\right\rangle(\phi(h))\right)$.

(ii) In the sense of bounded operators:

$$
\begin{aligned}
& P_{1} \ldots\left(P_{v} d f\left(H_{v}\right) Q_{v}+Q_{v} d f\left(H_{v}\right) P_{v}\right) \ldots P_{n} \\
& \quad=P_{1} \ldots\left(P_{v}\left[\frac{\overline{m(x, \hbar D)}}{i \hbar}, f\left(H_{v}\right)\right] Q_{v}+Q_{v}\left[\overline{\frac{m(x, \hbar D)}{i \hbar}}, f\left(H_{v}\right)\right] P_{v}\right) \ldots P_{n}+\mathcal{O}\left(\hbar^{2}\right) .
\end{aligned}
$$

Proof. (i) $m:=\chi_{\varepsilon}\left(h_{1}\right) \ldots \chi_{\varepsilon}\left(h_{n}\right) \tilde{m}$ for $\tilde{m}$ constructed in Corollary $8(* m$ continued to zero outside $U *)$. $m$ has values in $C_{0}^{\infty}\left(\mathbb{R}^{2 n}\right)$ and for $\eta<\varepsilon / 2$,

$$
\begin{aligned}
\chi_{\eta}\left(h_{1}\right) \ldots \chi_{\eta}\left(h_{n}\right)\left\{m, f\left(h_{v}\right)\right\} & =\chi_{\eta}\left(h_{1}\right) \ldots \chi_{\eta}\left(h_{n}\right) f^{\prime}\left(h_{v}\right)\left\{\tilde{m}, h_{v}\right\} \\
& =\chi_{\eta}\left(h_{1}\right) \ldots \chi_{\eta}\left(h_{n}\right)\left(d f\left(h_{v}\right)-\left\langle d f\left(h_{v}\right)\right\rangle(\phi(h))\right) .
\end{aligned}
$$

(ii) We suppress the closure of an operator in notation. By the calculus developed in Sect. 2 we have

$$
\begin{gathered}
f\left(H_{v}\right)=f\left(h_{v}\right)(x, \hbar D)+\hbar^{2} f_{2}(x, \hbar D)+\mathcal{O}\left(\hbar^{3}\right), \\
d f\left(H_{v}\right)=\left(d f\left(h_{v}\right)\right)(x, \hbar D)+\mathcal{O}\left(\hbar^{2}\right), \\
\chi_{\eta}\left(H_{v}\right)=\chi_{\eta}\left(h_{v}\right)(x, \hbar D)+\mathcal{O}\left(\hbar^{2}\right) .
\end{gathered}
$$


For the estimates it is sufficient to restrict all operators to $\mathscr{S}\left(\mathbb{R}^{n}\right)$ where we can use the Weyl calculus [Hör]. We have

$$
\begin{aligned}
{\left[m(x, \hbar D), f\left(H_{v}\right)\right]=} & \left(m \# f\left(h_{v}\right)-f\left(h_{v}\right) \# m\right)(x, \hbar D) \\
& +\hbar^{2}\left(m \# f_{2}-f_{2} \# m\right)(x, \hbar D)+\mathcal{O}\left(\hbar^{3}\right) \\
= & i \hbar\left\{m, f\left(h_{v}\right)\right\}(x, \hbar D)+\mathcal{O}\left(\hbar^{3}\right) .
\end{aligned}
$$

For $a_{i} \in S(1), A_{i}=a_{i}(x, \hbar D)+\mathcal{O}\left(\hbar^{2}\right)(i \in\{1, \ldots, n\})$ it holds

$$
A_{1} \ldots A_{n}=a_{1} \ldots a_{n}(x, \hbar D)+\frac{i \hbar}{2} \sum_{i<j}\left(\left\{a_{i}, a_{j}\right\} \prod_{i \neq k \neq j} a_{k}\right)(x, \hbar D)+\mathcal{O}\left(\hbar^{2}\right) \text {. }
$$

For $\eta$ from (i) we obtain

$$
\begin{aligned}
& \chi_{\eta}\left(H_{1}\right) \ldots \chi_{\eta}\left(H_{v}\right)\left[m(x, \hbar D), f\left(H_{v}\right)\right] \chi_{\eta}\left(H_{v+1}\right) \ldots \chi_{\eta}\left(H_{n}\right) \\
& \quad=i \hbar \chi_{\eta}\left(h_{1}\right) \ldots \chi_{\eta}\left(h_{n}\right)\left\{m, f\left(h_{v}\right)\right\}(x, \hbar D)+\hbar^{2} \text { second order }+\mathcal{O}\left(\hbar^{3}\right)
\end{aligned}
$$

and

$$
\begin{aligned}
& \chi_{\eta}\left(H_{1}\right) \ldots \chi_{\eta}\left(H_{v}\right) d f\left(H_{v}\right) \chi_{\eta}\left(H_{v+1}\right) \ldots \chi_{\eta}\left(H_{n}\right) \\
& \quad=\chi_{\eta}\left(h_{1}\right) \ldots \chi_{\eta}\left(h_{n}\right) d f\left(h_{v}\right)(x, \hbar D)+\hbar \text { first order }+\mathcal{O}\left(\hbar^{2}\right) .
\end{aligned}
$$

By (i),

$$
\begin{gathered}
\chi_{\eta}\left(H_{1}\right) \ldots \chi_{\eta}\left(H_{v}\right)\left(\left[\frac{m(x, \hbar D)}{i \hbar}, f\left(H_{v}\right)\right]-d f\left(H_{v}\right)\right) \chi_{\eta}\left(H_{v+1}\right) \ldots \chi_{\eta}\left(H_{n}\right) \\
=\chi_{\eta}\left(h_{1}\right) \ldots \chi_{\eta}\left(h_{n}\right)\left\langle d f\left(h_{v}\right)\right\rangle(\phi(h))(x, \hbar D)+\hbar \text { first order }+\mathcal{O}\left(\hbar^{2}\right) \\
=\chi_{\eta}\left(H_{1}\right) \ldots \chi_{\eta}\left(H_{n}\right)\left\langle d f\left(h_{v}\right)\right\rangle(\phi(H))+\hbar \text { first order }+\mathcal{O}\left(\hbar^{2}\right) .
\end{gathered}
$$

In each term of the symbols of "first" and "second order" there is a Poisson bracket with at least one $\chi_{\eta}\left(h_{v}\right)$ for some $v$. Hence they are delocalized in energy. Applying projections we get

$$
\begin{aligned}
& P_{1} \ldots P_{v}\left(\left[\frac{m(x, \hbar D)}{i \hbar}, f\left(H_{v}\right)\right]-d f\left(H_{v}\right)\right) Q_{v} P_{v+1} \ldots P_{n} \\
&= \hbar P_{1} \ldots P_{v} \text { first order } Q_{v} P_{v+1} \ldots P_{n}+\mathcal{O}\left(\hbar^{2}\right) \\
&= \hbar P_{1} \ldots P_{v} \chi_{\eta / 2}\left(H_{1}\right) \ldots \chi_{\eta / 2}\left(H_{v}\right) \text { first order } \\
& \times \chi_{\eta / 2}\left(H_{v+1}\right) \ldots \chi_{\eta / 2}\left(H_{n}\right) P_{v+1} \ldots P_{n} Q_{v}+\mathcal{O}\left(\hbar^{2}\right) \\
&= \mathcal{O}\left(\hbar^{2}\right) .
\end{aligned}
$$

The other equality is proven in the same manner.

In order to obtain information about $d P$ we make the assumption that the distance between eigenvalues of $H_{v}$ is of the order $\hbar$. This can actually be deduced from the assumptions already made $\mathrm{cf}$. [Ch]. 
We suppose (H5): For the reduced resolvents

$$
S_{v}\left(E_{\hbar}^{v}\right):=\lim _{\varepsilon \rightarrow 0}\left(f\left(H_{v}\right)-E_{\hbar}^{v}+i \varepsilon\right)^{-1} Q_{v}
$$

it holds: $\left\|S_{v}\left(E_{\hbar}^{v}\right)\right\|=\mathcal{O}(1 / \hbar)$.

From Theorem 9 we conclude

Corollary 10. Assume (H1),.., (H5). Then

Proof.

$$
d P=\left[\overline{\frac{m(x, \hbar D)}{i \hbar}}, P\right]+\mathcal{O}(\hbar) \text {. }
$$

By Corollary 4,

$$
d P=\sum_{v=1}^{n} P_{1} \ldots d P_{v} \ldots P_{n}
$$

$$
\begin{aligned}
d P= & \sum_{v=1}^{n} P_{1} \ldots \frac{1}{2 \pi i} \int_{\Gamma_{v}}\left(f\left(H_{v}\right)-z\right)^{-1} d f\left(H_{v}\right)\left(f\left(H_{v}\right)-z\right)^{-1} d z \ldots P_{n} . \\
& \left(f\left(H_{v}\right)-z\right)^{-1}=\frac{1}{f\left(E_{\hbar}^{v}\right)-z} P_{v}+S_{v}(z) Q_{v} \quad \text { with } S_{v} \text { analytic. }
\end{aligned}
$$

Hence

It follows

$$
\begin{aligned}
& -\frac{1}{2 \pi i} \int_{\Gamma_{v}}\left(f\left(H_{v}\right)-z\right)^{-1} d f\left(H_{v}\right)\left(f\left(H_{v}\right)-z\right)^{-1} d z \\
& =P_{v} d f\left(H_{v}\right) S_{v} Q_{v}+Q_{v} S_{v} d f\left(H_{v}\right) P_{v} .
\end{aligned}
$$

$$
\begin{aligned}
d P & =\sum_{v=1}^{n} P_{1} \ldots \frac{1}{2 \pi i} \int_{\Gamma_{v}}\left(f\left(H_{v}\right)-z\right)^{-1}\left[\frac{m(x, \hbar D)}{i \hbar}, f\left(H_{v}\right)\right]\left(f\left(H_{v}\right)-z\right)^{-1} d z \ldots P_{n} \\
& +\mathcal{O}(\hbar) \\
& =\sum_{v=1}^{n} P_{1} \ldots\left[\frac{m(x, \hbar D)}{i \hbar}, P_{v}\right] \ldots P_{n}+\mathcal{O}(\hbar)=\left[\frac{m(x, \hbar D)}{i \hbar}, P\right]+\mathcal{O}(\hbar) \cdot \square
\end{aligned}
$$

We cite from [Ch2] the following statement:

Theorem 11. Assume (H1),.., (H4). For $a \in S(1)$ and a sequence of joint eigenstates $\psi_{\hbar}$ with $H_{v} \psi_{\hbar}=E_{\hbar}^{v} \psi_{\hbar}$, it holds:

$$
\lim _{\hbar \rightarrow 0}\left\langle\psi_{\hbar}, \overline{a(x, \hbar D)} \psi_{\hbar}\right\rangle=\langle a\rangle\left(\phi\left(E_{0}\right)\right) .
$$

The result concerning the limit of the quantum two form is

Theorem 12. Assume (H1),..,(H5). Then

$$
\lim _{\hbar \rightarrow 0} \frac{\hbar}{i} \frac{1}{\operatorname{tr} P} \operatorname{tr} P d P d P=\frac{1}{2}\langle\{m, m\}\rangle\left(\phi\left(E_{0}\right)\right) \text { at } \kappa=0 \text {. }
$$

Proof. Define $M:=\frac{\overline{m(x, \hbar D)}}{i \hbar}$. By Corollary 10,

$$
\operatorname{tr} P d P d P=\operatorname{tr} P([M, P][M, P]+\mathcal{O}(1))=-\operatorname{tr} P(M M+\mathcal{O}(1)) .
$$


By the Weyl calculus

$$
\frac{1}{\operatorname{tr} P} \operatorname{tr} P d P d P=\frac{i}{2 \hbar} \frac{1}{\operatorname{tr} P} \operatorname{tr} P \overline{\{m, m\}(x, \hbar D)}+\mathcal{O}(1),
$$

and the assertion is implied by Theorem 11 .

Let us now interpret the result.

We are interested in the limit of $\operatorname{tr} P d P d P$ at the point $\kappa=0$. The geometric objects used should be understood as local portions of objects which are non-trivial in general.

Recall the "quantum" geometry.

For a vicinity $V$ of $\kappa=0, \bigcup_{\kappa \in V}\left\langle\kappa, \operatorname{Ran} P_{\kappa}\right\rangle$ is a complex vector bundle embedded in $V \times L^{2}\left(\mathbb{R}^{n}\right)$, where a natural connection respecting the subbundle is given $(* Q:=1-P *)$ by

$$
P d P+Q d Q=d-[(d P), P] .
$$

So $[(d P), P]$ generates parallel - adiabatic - motion.

On the subbundle the connection reduces to $\nabla=P d$ whose curvature is the operator-valued two form on $V$ acting on sections by

$$
1 / 2[d-(d P), d-(d P)]=1 / 2[(d P),(d P)]=(d P)(d P) .
$$

So tr $P d P d P$ represents the first Chern class of the bundle.

It was first recognized by [S] that for $\operatorname{dim} P=1$ Berry's Phase is the holonomy of such a bundle.

For details of the description of the "classical" geometry we refer to [M]. If there exist actions $\phi_{v} \circ h$ which are smooth in the parameters they can always be chosen such that $\left\langle d \phi_{v} \circ h\right\rangle \equiv 0$. Clearly it is then possible to find a smooth $m$ such that

The vector fields

$$
\left\{m, \phi_{v} \circ h\right\}=d \phi_{v} \circ h \quad \text { all } v,\langle m\rangle \equiv 0 \text {. }
$$

$$
\frac{\partial}{\partial \kappa^{k}}+X_{m_{k}} \quad k \in\{1, \ldots, d\}
$$

span the horizontal distribution on $U_{0} \times \mathbb{R}^{2 n}$ defined by averaging $\frac{\partial}{\partial \kappa^{k}}$ over the toral action given by the joint flow of $\phi \circ h$. They project to a connection on $\bigcup_{\kappa \in U_{0}}\left\langle\kappa,(\phi \circ h(\kappa))^{-1}\left(\phi\left(0, E_{0}\right)\right)\right\rangle$ which is a candidate of an "adiabatic subbundle."

Let now $\gamma$ be a rectifiable curve in $U_{0}$ and $W$ be unitary with

$$
\frac{d}{d t} W(t)=\frac{m(\gamma(t))\left(\gamma^{\prime}(t)\right)(x, \hbar D)}{i \hbar} W(t), \quad W(0)=1 .
$$

By Corollary 10,

$$
\begin{aligned}
\frac{d}{d t}( & P(\gamma(t)) W(t)-W(t) P(0)) \\
& =\frac{m(\gamma(t))\left(\gamma^{\prime}(t)\right)(x, \hbar D)}{i \hbar}(P(\gamma(t)) W(t)-W(t) P(0))+\mathcal{O}(\hbar) .
\end{aligned}
$$


It follows

$$
P(\gamma(t)) W(t)-W(t) P(0)=\mathcal{O}(\hbar)
$$

So $\frac{m(x, \hbar D)}{i \hbar}$ generates quantum adiabatic motion up to a semiclassical small error.

The curvature of the classical connection turns out to be $\frac{1}{2}\langle\{m, m\}\rangle$. Hence the content of Theorem 12 can be stated as:

$$
\frac{\hbar}{i} \times \text { mean quantum curvature } \rightarrow \text { classical curvature } \quad(\hbar \rightarrow 0) \text {. }
$$

The two forms used in $[\mathrm{H}]$ and [B2] to compute the Hannay angles are coordinate versions of $1 / 2\langle\{m, m\}\rangle$. In this sense the result was derived by Berry [B2].

\section{Non-Integrable Systems}

As it was proposed by [Se, S-T] one might consider a smoothed out quantum two form which admits an asymptotic expansion without any assumptions on the classical flow. This works if the classical Hamiltonian satisfies the conditions for selfadjointness and differentiability, and if the portion of phase space corresponding to the energies considered is compact in phase space.

Fix a classical energy $E_{0} \in \mathbb{R}^{+}$and $E_{1}<E_{0}<E_{2}$. Consider $\chi_{i} \in C_{0}^{\infty}(\mathbb{R})$ $\left(i \in\{1, \ldots, 4\}\right.$ such that $\operatorname{supp} \chi_{i} \subset\left(E_{1}, E_{2}\right), \chi_{i} \equiv 1$ on $\left[E_{1}+\varepsilon, E_{2}-\varepsilon\right]$ (some $\varepsilon>0$ ).

The statement is

Theorem 13. Assume (H1), (H2) on h and compactness of $h^{-1}\left(\left[E_{1}, \mathrm{E}_{2}\right]\right) \subset \mathbb{R}^{2 n}$. Then: $\chi_{1}(H) d \chi_{2}(H) d \chi_{3}(H) \chi_{4}(H)$ is trace class and there exist $a_{i} \in C_{0}^{\infty}\left(\mathbb{R}^{2 n}\right)\left(i \in \mathbb{N}_{0}\right)$ such that for $N \in \mathbb{N}_{0}$,

$$
\hbar^{n} \operatorname{tr}\left(\chi_{1}(H) d \chi_{2}(H) d \chi_{3}(H) \chi_{4}(H)\right)=\sum_{i=0}^{N} \hbar^{i} \frac{1}{(2 \pi)^{n}} \int_{\mathbb{R}^{2 n}} a_{i}+\hbar^{N+1} \mathcal{O}(1) .
$$

Proof. It holds for an $N \in \mathbb{N}$ (cf.: [Hör])

$$
\hbar^{n}\|a(x, \hbar D)\|_{\mathrm{tr}} \leqq \text { const } \sup _{\substack{\alpha, \beta \in \mathbb{N}_{0}^{2 n} \\|\alpha|+|\beta| \leqq N}}\left\|p^{\alpha} D^{\beta} a\right\|_{L^{2}\left(\mathbb{R}^{2 n}\right)}
$$

and for $a(x, \hbar D)$ trace class and $a$ integrable

$$
\hbar^{n} \operatorname{tr} a(x, \hbar D)=\frac{1}{(2 \pi)^{n}} \int_{\mathbb{R}^{2 n}} a
$$

Pick now $\chi \in C_{0}^{\infty}(\mathbb{R})$, supp $\chi \subset\left(E_{1}, E_{2}\right), \chi \equiv 1$ on supp $\chi_{1}$. By a result of $[\mathrm{H}-\mathrm{R}]$

$$
\left\|\hbar^{n} \chi(H)\right\|_{\mathrm{tr}}=\mathcal{O}(1),
$$


furthermore there are $\chi^{i} \in C_{0}^{\infty}\left(\mathbb{R}^{2 n}\right)\left(i \in \mathbb{N}_{0}\right)$ such that for $N \in \mathbb{N}_{0}$,

and $\left\|\hbar^{n} R_{N \chi}\right\|_{\mathrm{tr}}=\mathcal{O}(1)$.

$$
\chi(H)=\sum_{i=0}^{N} \hbar^{i} \chi^{i}(x, \hbar D)+\hbar^{N+1} R_{N \chi}
$$

Theorem 5 and Weyl calculus imply: There exist $a_{i} \in C_{0}^{\infty}\left(\mathbb{R}^{2 n}\right)\left(i \in \mathbb{N}_{0}\right)$ such that for $N \in \mathbb{N}_{0}$

$$
\chi_{1}(H) d \chi_{2}(H) d \chi_{3}(H) \chi_{4}(H)=\sum_{i=0}^{N} \hbar^{i} a_{i}(x, \hbar D)+\hbar^{N+1} R_{N}
$$

with $R_{N}$ bounded in operator norm uniformly in $\hbar$. Application of $\chi(H)$ gives:

$$
\begin{aligned}
\chi_{1}(H) d \chi_{2}(H) d \chi_{3}(H) \chi_{4}(H)= & \chi(H) \chi_{1}(H) d \chi_{2}(H) d \chi_{3}(H) \chi_{4}(H) \\
= & \sum_{i=0}^{N} \hbar^{i} \chi^{i}(x, \hbar D) \sum_{i=0}^{N} \hbar^{i} a_{i}(x, \hbar D) \\
& +\hbar^{N+1}\left[R_{N \chi} \sum_{i=0}^{N} \hbar^{i} a_{i}(x, \hbar D)+\chi(H) R_{N}\right] .
\end{aligned}
$$

Clearly $\hbar^{n}\left\|R_{N \chi} \sum_{i=0}^{N} \hbar^{i} a_{i}(x, \hbar D)+\chi(H) R_{N}\right\|_{\mathrm{tr}} \leqq \operatorname{const}(\hbar \rightarrow 0)$.

As the symbols of the explicit terms are all supported in $h^{-1}\left(\left(E_{1}, E_{2}\right)\right)$ one applies the Weyl calculus for the flat metric with conformal factor $\frac{\text { const }}{1+p^{2}}\left(p \in \mathbb{R}^{2 n}\right)$ to conclude

$$
\hbar^{n}\left\|\sum_{i=0}^{N} \hbar^{i} \chi^{i}(x, \hbar D) \sum_{i=0}^{N} \hbar^{i} a_{i}(x, \hbar D)-\sum_{i=0}^{N} \hbar^{i} a_{i}(x, \hbar D)\right\|_{\mathrm{tr}} \leqq \hbar^{N+1} \text { const } \quad(\hbar \rightarrow 0)
$$

So $\chi_{1}(H) d \chi_{2}(H) d \chi_{3}(H) \chi_{4}(H)$ is trace class and the expansion holds.

We remark that for $\chi_{1}=\ldots=\chi_{4}=: \chi$ the leading term is

$$
\frac{\hbar}{i(2 \pi)^{n}} \int_{\mathbb{R}^{2 n}} \chi^{2}(h) \frac{1}{2}\{d \chi(h), d \chi(h)\} \text {. }
$$

The asymptotic expansion stated in Theorem 13 is essentially a special case of Proposition 7-1 of [S-T]. The following points in the Schrader-Taylor approach are different:

The Hamiltonians considered are second order elliptic operators on a compact manifold with additional gauge fields. The functional calculus is based on an analysis of the fundamental solution of the wave equation rather than complex powers of the Hamiltonian. Questions related to differentiability with respect to the external parameters are not explicitly treated.

Acknowledgement. I should like to thank R. Seiler for pointing the subject out to me and for discussions. 


\section{References}

[A-S-Y] Avron, J.E., Seiler, R., Yaffe, L.G.: Adiabatic theorems and applications to the quantum hall effect. Commun. Math. Phys. 82, 33-49 (1987)

[B] Berry, M.V.: Quantal phase factors accompanying adiabatic changes. Proc. R. Soc. Lond. A392, 45-57 (1984)

[B2] Berry, M.V.: Classical adiabatic angles and quantal adiabatic phase. J. Phys. A18, 15-27 (1985)

[Ch] Charbonnel, A.M.: Comportement semi-classique du spectre conjoint d'opérateurs pseudodifférentiels qui commutent. Asymptotic Anal. 1, 227-261 (1988)

[Ch2] Charbonnel, A.M.: Comportement semi-classique des systèmes ergodiques, Preprint, Université de Nantes (1989)

[Kn] Golin, S., Knauf, A., Marmi, S.: The Hannay angles: geometry, adiabaticity, and an example. Commun. Math. Phys. 123, 95-122 (1989)

[H] Hannay, J.H.: Angle variable holonomy in adiabatic excursion of an integrable Hamiltonian. J. Phys. A 18, 221-230 (1985)

[H-R] Helffer, B., Robert, D.: Calcul fonctionelle par la transformation de Mellin et opérateurs admissibles. J. Funct. Anal. 53, 246-268 (1983)

[H-M-R] Helffer, B., Martinez, A., Robert, D.: Ergodicité et limite semi-classique, Commun. Math. Phys. 109, 313-326 (1987)

[Hör] Hörmander, L.: The Weyl calculus of pseudo-differential operators. Commun. Pure Appl. Math. 32, 359-443 (1979)

[K] Kato, T.: On the adiabatic theorem in quantum mechanics. J. Phys. Soc. Jpn. 5, 435-439 (1950)

[M-T] Mead, C.A., Truhlar, D.G.: On the determination of Born-Oppenheimer nuclear motion wave functions including complications due to conical intersections and identical nuclei. J. Chem. Phys. 70, 2284-2296 (1979)

[M] Montgomery, R.: The connection whose holonomy is the classical adiabatic angles of Hannay and Berry and its generalisation to the non-integrable case, Commun. Math. Phys. 120, 269-294 (1988)

[S-T] Schrader, R., Taylor, M.: Semiclassical asymptotics, gauge fields, and quantum chaos. J. Funct. Anal. 83, 258-316 (1989)

[Se] Seiler, R.: Private communication

[S] Simon, B.: Holonomy, the quantum adiabatic theorem and Berry's phase. Phys. Rev. Lett. 51, 2167-2170 (1983)

Communicated by B. Simon

Received August 3, 1989; in revised form September 28, 1989 
